\title{
Relationship of Serum Bicarbonate Levels with 1-Year Graft Function in Kidney Transplant Recipients in Switzerland
}

\author{
Anna Wiegand ${ }^{a} \quad$ Nicole Graf ${ }^{b}$ Marco Bonani ${ }^{a}$ Diana Frey ${ }^{c}$ \\ Rudolf P. Wüthrich ${ }^{a}$ Nilufar Mohebbia \\ ${ }^{a}$ Division of Nephrology, University Hospital Zurich, Zurich, Switzerland; b Graf Biostatistics, \\ Winterthur, Switzerland; ' Division of Rheumatology, University Hospital Zurich, Zurich, \\ Switzerland
}

\section{Keywords}

Metabolic acidosis $\cdot$ Outcome $\cdot$ Renal function

\begin{abstract}
Background: Metabolic acidosis (MA) is common in kidney transplant recipients (KTRs). Several studies have shown that MA is involved in the progression of chronic kidney disease. However, it is unclear if there is also a relationship between serum bicarbonate and graft function after kidney transplantation (KTx). We hypothesized that low serum bicarbonate is associated with a lower estimated glomerular filtration rate (eGFR) 1 year after KTx. Methods: We performed a post hoc analysis of a single-center, open-label randomized trial in 90 KTRs and investigated the relationship of serum bicarbonate and graft function in the first year after KTx. Results: Prevalence of MA was high after KTx (63\%) and decreased to $28 \%$ after 1 year. Bicarbonate $(20.6 \pm 3.0$ to $22.7 \pm 2.7 \mathrm{mmol} / \mathrm{L})$ increased in the first year after transplantation whereas eGFR ( $53.4 \pm 15.8$ to $\left.56.9 \pm 18.5 \mathrm{~mL} / \mathrm{min} / 1.73 \mathrm{~m}^{2}\right)$ did not change significantly. Higher serum bicarbonate $(p=0.029)$ was associated with higher eGFR in the first year after KTx. Conclusion: Prevalence of MA is high in KTRs. In the first year after KTx, serum bicarbonate was positively correlated with eGFR, suggesting a potential role of MA in kidney graft function.
\end{abstract}

(c) 2019 The Author(s)

Published by S. Karger AG, Basel

\section{Introduction}

Metabolic acidosis (MA), usually defined as a low $\mathrm{pH}$ and low serum bicarbonate levels $(<22 \mathrm{mmol} / \mathrm{L})$, is a typical complication in kidney transplant recipients (KTRs), with a prevalence of $12-58 \%$ [1]. Compared to chronic kidney disease (CKD) patients with similar renal 


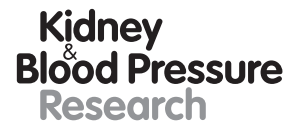

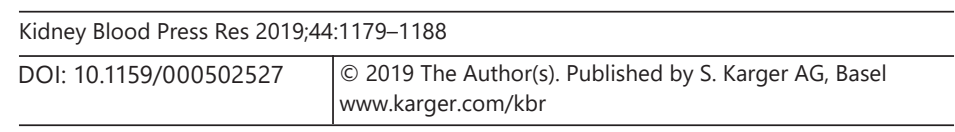

Wiegand et al.: Serum Bicarbonate in KTR

function, MA seems to be consistently more common and more severe in KTRs [1-3]. Specifically, in the time directly after transplantation, MA is frequent because of calcineurin inhibitor nephrotoxicity, acute rejection, suboptimal allograft function, and ischemic tubular dysfunction [3,4]. Among these factors, donor-specific conditions like age and the type of transplant might play an important role [1].

MA has been associated with a variety of complications such as anemia, uremic bone disease, protein-energy wasting, increased inflammatory mediators, insulin resistance, and the impairment of myocardial function as well as disturbances in mineral metabolism, growth hormone production, and thyroid gland function [5-8]. Additionally, acidosis and transplantation are associated with an increased risk of bone disturbances [9].

Observational studies have shown that MA is associated with increased mortality in both dialysis patients and patients with nondialysis-dependent CKD [10]. Furthermore, over the past few years, it has been reported that MA may play an important role in the progression of CKD and that low serum bicarbonate levels are associated with a higher risk of progressing to end-stage renal disease [6,10-15]. Consequently, the treatment of MA in CKD patients with alkalinizing agents or fruit and vegetables has been found to delay the progression of glomerular filtration rate (GFR) loss [16-19].

Recently, a first retrospective cohort study evaluated the prevalence, longitudinal trends, and risk factors for MA in KTRs [20], but there were several limitations. First, only one-quarter of the grafts were from deceased donors. Second, the cohort was comparatively healthy, with a low number of graft losses in the observed time period.

We wanted to test the association of serum bicarbonate levels and graft function in a representative transplantation cohort in Switzerland. We hypothesized that low serum bicarbonate is associated with a lower estimated (e)GFR 1 year after kidney transplantation (KTx).

\section{Methods}

\section{Patients and Study Design}

This study is the post hoc analysis of a previously conducted single-center, parallelgroup, open-label, randomized clinical trial performed in an academic setting at the University Hospital Zurich (Switzerland) that tested denosumab for its effects on bone mineral density in 90 KTRs. Details of the POSTOP (Prolia for Osteoporosis of Transplant Operated Patients) study were reported previously $[21,22]$. The study was designed and conducted according to the International Conference on the Harmonization of Good Clinical Practice standards, approved by the local Ethics Committee (IRB approval No. 2011-0032), and registered on ClinicalTrials.gov (NCT01377467).

Briefly, we recruited a total of 90 KTRs $>18$ years that had undergone KTx $<28$ days prior to examine the changes in bone mineral density over 1 year. Although half of the patients were treated with denosumab, all patients were included in this post hoc analysis since there is no evidence of any direct effects of denosumab on acid-base homeostasis. Nonetheless, we corrected all models for the randomization group to eliminate potential effects of denosumab on the outcome. Patients received standard triple immunosuppression including a calcineurin inhibitor (cyclosporine or tacrolimus), mycophenolate, and steroids. Induction treatment was performed with basiliximab or antithymocyte globulin according to the standard care protocol for KTRs at our center [21].

We investigated the relationship of serum bicarbonate levels and graft function in the first year after KTx. MA was defined as a serum bicarbonate level $<22 \mathrm{mmol} / \mathrm{L}$. Graft function was determined by eGFR according to the 2009 CKD-EPI creatinine equation. Values were set to missing if patients had been treated for MA 1 month before the measurement (if not 
Table 1. Baseline and 1-year characteristics of the entire cohort

\begin{tabular}{|c|c|c|c|}
\hline & At baseline & At 1 year & $p$ value \\
\hline Total number of patients & 90 & 90 & - \\
\hline Mean age, years (SD) & $49.5(13.4)$ & - & - \\
\hline Male & $57(63.3)$ & - & - \\
\hline \multicolumn{4}{|l|}{ Ethnicity } \\
\hline Caucasian & $86(95.6)$ & - & - \\
\hline Asian & $2(2.2)$ & - & - \\
\hline African & $1(1.1)$ & - & - \\
\hline Other & $1(1.1)$ & - & - \\
\hline \multicolumn{4}{|l|}{ ESRD diagnosis } \\
\hline Chronic glomerulonephritis & $30(33.3)$ & - & - \\
\hline Diabetic nephropathy & $7(7.8)$ & - & - \\
\hline Vascular nephropathy & $8(8.9)$ & - & - \\
\hline Polycystic kidney disease & $20(22.2)$ & - & - \\
\hline Other hereditary diseases & $5(5.6)$ & - & - \\
\hline Other & $20(22.2)$ & - & - \\
\hline Deceased donor & $44(48.9)$ & - & - \\
\hline Graft loss & $1(1.1)$ & - & - \\
\hline Rejection & $8(8.9)$ & & \\
\hline Repeat transplantation & $14(15.6)$ & - & - \\
\hline Randomization: denosumab & $46(51.1)$ & - & - \\
\hline \multicolumn{4}{|l|}{ Dialysis mode } \\
\hline Hemodialysis & $57(63.3)$ & - & - \\
\hline Peritoneal dialysis & $16(17.8)$ & - & - \\
\hline Pre-emptive transplantation & $17(18.9)$ & - & - \\
\hline Cyclosporine & $26(28.9)$ & $19(21.3)$ & - \\
\hline Tacrolimus & $64(71.1)$ & $70(78.7)$ & - \\
\hline Induction therapy $^{1}$ & 89 (98.9) & - & - \\
\hline Mean body mass index (SD) & $25.7(5.0)$ & $25.8(4.8)$ & 0.287 \\
\hline Mean eGFR, $\mathrm{mL} / \mathrm{min} / 1.73 \mathrm{~m}^{2}$ (SD) & $53.3(15.8)$ & $56.9(18.5)$ & 0.303 \\
\hline Mean bicarbonate, mmol/L (SD) & $20.6(3.0)$ & $22.7(2.6)$ & $<0.001$ \\
\hline Mean sodium, mmol/L (SD) & $138.1(3.7)$ & $139.3(2.8)$ & 0.048 \\
\hline Mean chloride, mmol/L(SD) & $105.9(4.2)$ & $104.6(2.9)$ & 0.022 \\
\hline Mean calcium, mmol/L (SD) & $2.3(0.2)$ & $2.5(0.2)$ & $<0.001$ \\
\hline Mean phosphate, mmol/L (SD) & $0.6(0.2)$ & $0.9(0.3)$ & $<0.001$ \\
\hline Mean magnesium, mmol/L (SD) & $0.6(0.1)$ & $0.7(0.1)$ & 0.001 \\
\hline Mean PTH, ng/L (SD) & $156.2(145.8)$ & $105.5(75.6)$ & $<0.001$ \\
\hline
\end{tabular}

All values are displayed as $n(\%)$, if not otherwise stated. The following values were set to missing for 1 patient who was treated for metabolic acidosis 1 month before measurement at baseline and for 4 patients at 1 year: body mass index, PTH, calcium, phosphate, eGFR, bicarbonate, magnesium, sodium, and chloride.

${ }^{1}$ Patients received basiliximab (68.9\%) or antithymocyte globulin $(30.0 \%)$.

otherwise indicated). Blood samples were taken at baseline (on average 15 days after KTx) and at 3,6 , and 12 months thereafter.

\section{Statistical Analyses}

eGFR and bicarbonate differences between baseline and 12 months were compared with the Wilcoxon signed-rank test. eGFR was analyzed using a linear mixed model with random intercept. Treatment group (denosumab) versus control group (no denosumab), age at time of transplantation, donor status (living vs. deceased), and bicarbonate (not set to missing for 


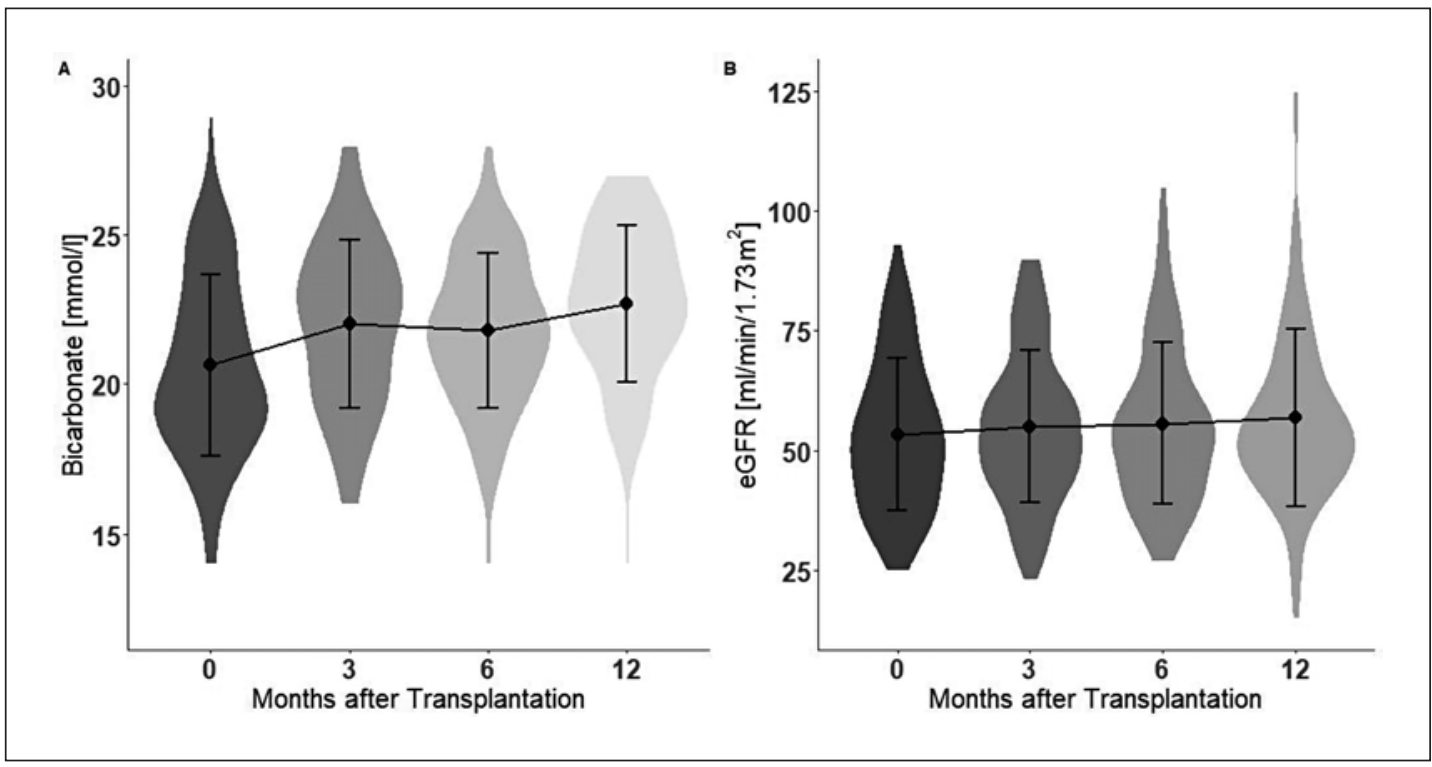

Fig. 1. Bicarbonate (A) and eGFR (B) (CKD-EPI 2009) at months $0,3,6$, and 12 . For months $0,3,6,12$ there were 87, 59, 72, and 75 valid data for bicarbonate and 89, 85, 87, and 84 for eGFR (CKD-EPI 2009), respectively. Values were set to missing for patients treated with alkali therapy. Errors bars show the standard deviation.

patients treated with alkali therapy) were included as fixed effects. The restricted maximum likelihood method was used to estimate the parameter values of the linear mixed model. The comparison between a random intercept model and a random intercept and random slope (for bicarbonate) model indicated that the random intercept and random slope model did not perform better. Assumptions of the within-group error were checked with plots of the standardized residuals versus fitted values and a $Q-Q$ plot of the standardized residuals. Assumptions of the random effects were assessed by a Q-Q plot of the random effects. All analyses were performed in the R programming language v3.3.3 (R Core Team, 2017) and SPSS v20 (IBM Corp.). The nlme package [23] was used to run the linear mixed model.

\section{Results}

\section{Baseline Characteristics}

Demographic and baseline characteristics of the study population are presented in Table 1. The mean age of the study cohort was $49.5 \pm 13.4$ years and $57(63 \%)$ were male. The majority were undergoing KTx for the first time (84\%) and were of Caucasian origin (96\%). Most patients were on renal replacement therapy before KTx (81\%) and 17 (19\%) underwent pre-emptive transplantation. Maintenance immunosuppression consisted of calcineurin inhibitor (tacrolimus $71 \%$ or cyclosporine $29 \%$ ) combined with mycophenolate and prednisone. Half of the patients were treated with denosumab as part of the POSTOP study protocol [21]; 44/90 kidney transplants (49\%) were from deceased donors. Mean eGFR at baseline was $53.3 \pm 15.8 \mathrm{~mL} / \mathrm{min} / 1.73 \mathrm{~m}^{2}$ and mean bicarbonate was $20.6 \pm 3.0 \mathrm{mmol} / \mathrm{L}$.

Prevalence of MA Decreased in the First Year after KTX

Serum bicarbonate levels increased continuously from $20.6 \pm 3.0 \mathrm{mmol} / \mathrm{L}$ at baseline (11 \pm 8 days after KTx) to $22.7 \pm 2.6 \mathrm{mmol} / \mathrm{L}(p<0.001)$ in the first year after KTx (Fig. 1a). In 
Fig. 2. Prevalence of metabolic acidosis over the course of 1 year after kidney transplantation in all patients.

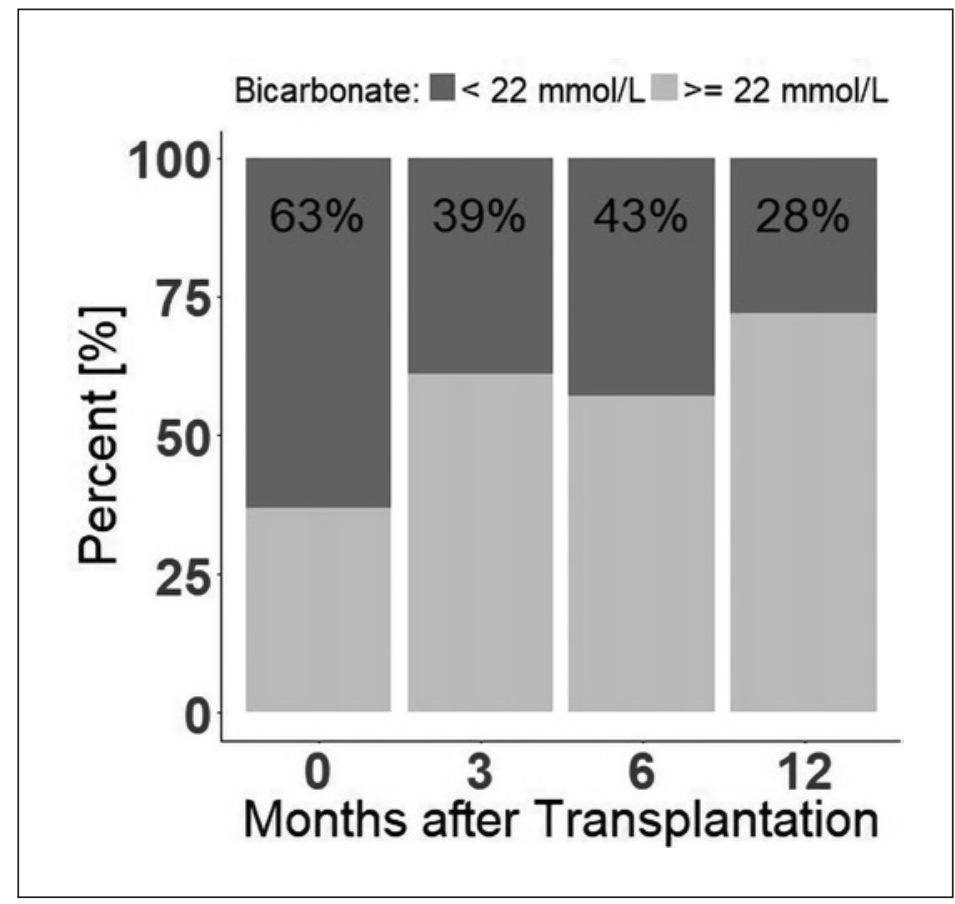

parallel, eGFR levels had increased numerically from $53.3 \pm 15.8$ to $56.9 \pm 18.5 \mathrm{~mL} / \mathrm{min}(p=$ 0.303) 1 year after KTx (Fig. 1b).

The prevalence of MA, defined as serum bicarbonate $<22 \mathrm{mmol} / \mathrm{L}$, was highest directly after KTx (63\%) and declined over the first year post-KTx to 28\% (Fig. 2). There was no difference in the prevalence of MA depending on the respective calcineurin inhibitor included in the immunosuppressive regimen of the study participants.

\section{Higher Serum Bicarbonate Levels Are Associated Positively with eGFR within the First} Year after KTX

In Figure 3, eGFR and serum bicarbonate levels of each individual patient at different time points are shown. Interestingly, we observed different patterns and strong interindividual variations in the eGFR and bicarbonate profiles in the first year after KTx. Additionally, a heat map indicated, by darker coloring, the regions with the highest density of values (bicarbonate and the corresponding eGFR) (Fig. 4).

A linear mixed model with random intercept adjusted for age, serum bicarbonate levels, donor status (living vs. deceased), and randomization (POSTOP trial) was used for further analysis. Higher serum bicarbonate levels $(p=0.029)$ were associated with higher eGFR values within the first year after KTx. Randomization $(p=0.786)$ of the POSTOP trial, younger age $(p=0.063)$, and donor status ( $p=0.821)$ were not associated with eGFR over the first year after KTx.

\section{Discussion}

MA is a frequent condition in CKD and has been associated with various clinical complications. Recently, the impact of MA on the progression of kidney function in CKD patients and the beneficial effect of alkali therapy on CKD progression has gained in importance. Interest- 


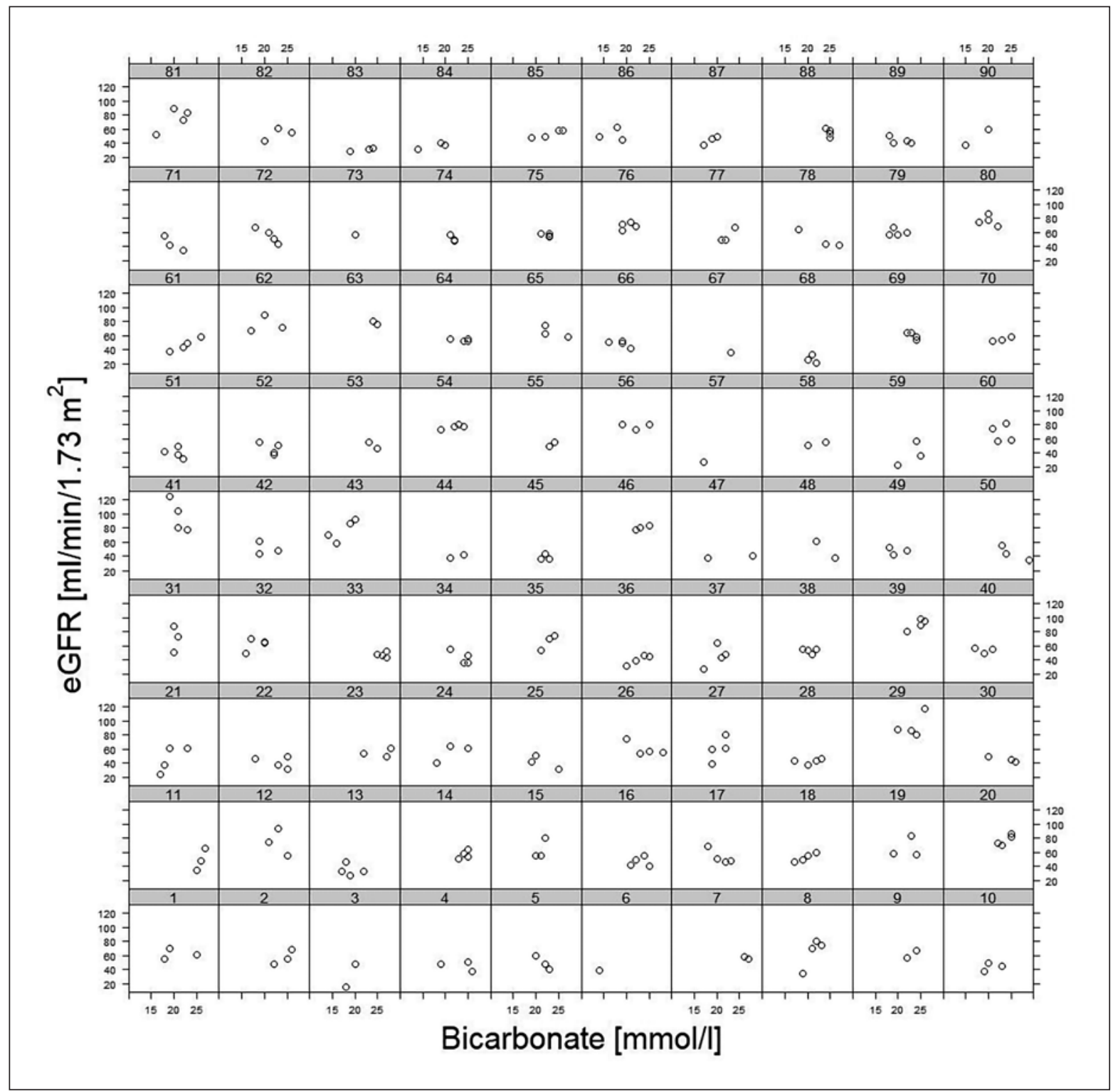

Fig. 3. eGFR (CKD-EPI 2009) versus serum bicarbonate at months 0, 3, 6, and 12 after kidney transplantation in individual patients, demonstrating strong interindividual patterns of eGFR trajectory.

ingly, despite the high prevalence of MA in KTRs, only few data exist regarding the impact of MA on graft outcome after KTx. We performed a post hoc analysis of a previously performed single-center, parallel-group, open-label, randomized clinical trial [21], and investigated the relationship between serum bicarbonate levels and eGFR over the first year after KTx. Our study presents evidence that serum bicarbonate levels are positively associated with eGFR in the first year after KTx.

In our study, the prevalence of MA, defined as serum bicarbonate $<22 \mathrm{mmol} / \mathrm{L}$, was highest after KTx (63\%) and declined over the first year post-KTx to $28 \%$. According to the literature, the prevalence of MA after KTx ranges from 11 to $58.1 \%$ and occurs at higher eGFR levels than in CKD patients [1]. The prevalences reported in our cohort were highly concordant with previously published results. Messa et al. [1] reviewed 11 studies in which the preva- 
Fig. 4. Scatter-plot depicting the correlation of eGFR (CKD-EPI 2009) and serum bicarbonate over the first year after kidney transplantation in all patients. Darker areas highlight regions with the highest density of values.

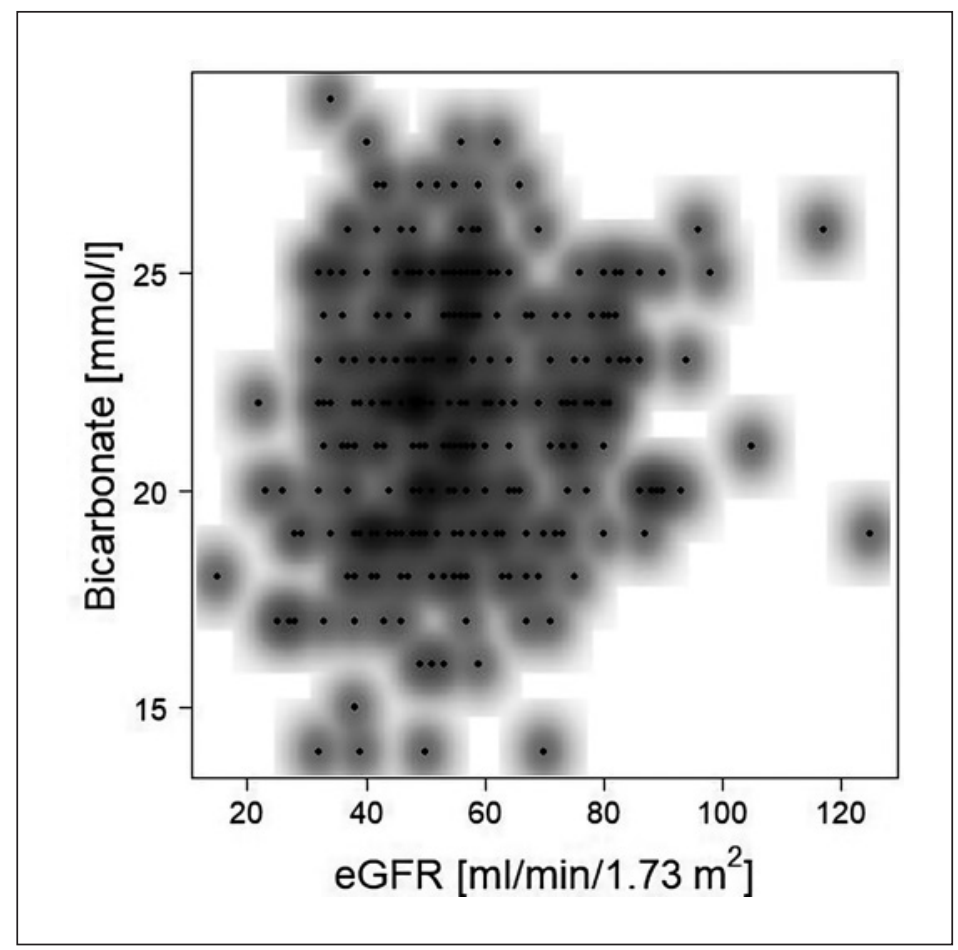

lence of MA in KTRs was reported as between 11 and 58\%. A large variation in prevalences across studies is mainly due to different study populations and definitions of MA. Interestingly, a recent study by Park et al. [20] described a prevalence of MA of only $10 \% 1$ year after KTx in a cohort of 2,318 adult Korean KTRs. However, compared to our cohort, the number of transplants from deceased donors was considerably lower (48 and $24 \%$, respectively), which could explain the difference in the occurrence of MA. Due to the lack of larger, solid studies, it is not yet verified if donor characteristics (e.g., a living vs. a deceased donor) play a role in the incidence of MA after KTx. Nonetheless, in our study, the prevalence of MA was highest directly after KTx and further declined over the first year. This may indicate that donor-specific factors play a role in the development of MA, as previously stated by Messa et al. [1].

As expected, bicarbonate levels increased within the first year after KTx in our cohort whereas for eGFR no significant change was observed. Reduction of medication, particularly immunosuppression, and general stabilization of the kidney graft function may be the most important underlying causes for the increase in bicarbonate levels. Interestingly, in our cohort, the individual course of eGFR varied strongly between the 90 study participants. This strong variability has been previously reported [24,25] and is not surprising, as many factors such as medication, donor characteristics, and general health status differ strongly not only between patients but also for the individual patient, and for medication. However, high variability of kidney function after KTx has been described as an independent risk factor for graft loss and death [24-26]. Thus, we used a mixed linear model including all available eGFR and bicarbonate values over the first year after KTx. In our analysis, higher serum bicarbonate levels were associated with higher eGFR within the first year after KTx. Importantly, our study is the first to include Caucasian kidney recipients that were treated according to a European standard transplant program, and it confirms previously published data on a Korean cohort by Park et al. [20]. 


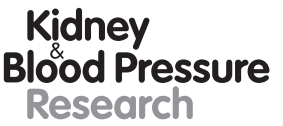

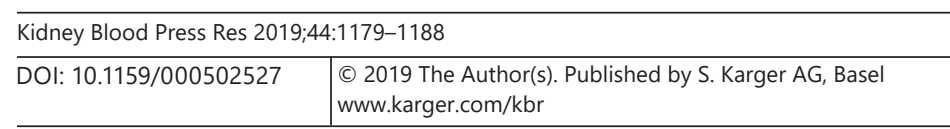

Wiegand et al.: Serum Bicarbonate in KTR

Several studies have reported that eGFR levels at 1 year after KTx are a strong predictor of long-term transplant outcomes [27-33]. Considering the positive association between bicarbonate and GFR levels, correction of MA in KTRs may have the potential to improve eGFR levels and, subsequently, the long-term graft outcomes. However, further randomized controlled interventional trials are required to demonstrate the beneficial effect of alkali therapy on transplant outcome.

Our study has several limitations. First, there was a relatively small number of participants, and serum bicarbonate measurements were not performed at each transplant visit. Furthermore, additional confounders emanating from the post hoc study design may have been present. Nonetheless, this design can also be seen as a strength, since study visits were scheduled prospectively according to the study protocol and patients were all seen at the same transplant center, producing high-quality data on a representative Swiss cohort. Finally, because of the study design, the results did not allow any conclusions regarding a causal relationship between serum bicarbonate and eGFR.

In conclusion, MA is highly prevalent in KTRs and serum bicarbonate values are associated with eGFR levels in the first year after KTx, suggesting a potential role of MA on kidney graft function. A randomized controlled interventional study would be desirable to prove a beneficial effect of alkali therapy on graft function in KTRs with MA.

\section{Statement of Ethics}

The research was conducted ethically in accordance with the World Medical Association Declaration of Helsinki. Subjects (or their parents or guardians) gave their written informed consent and the study protocol was approved by the institute's committee on human research.

\section{Disclosure Statement}

The authors declare no conflicts of interest.

\section{Funding Sources}

No funding was received for this project.

\section{Author Contributions}

Research idea and design: AW, NM, RPW; data acquisition: AW, NM, MB, RPW, DF; statistical data analysis: NG; data interpretation: AW, NM; writing of the manuscript: AW, NM, NG. Each author contributed important intellectual content during manuscript drafting and accepts accountability for the overall work. 


\begin{tabular}{l|l}
\hline \multicolumn{2}{l}{ Kidney Blood Press Res 2019;44:1179-1188 } \\
\hline DOI: 10.1159/000502527 & $\begin{array}{l}\text { @ 2019 The Author(s). Published by S. Karger AG, Basel } \\
\text { www.karger.com/kbr }\end{array}$ \\
\hline
\end{tabular}

Wiegand et al.: Serum Bicarbonate in KTR

\section{References}

1 Messa PG, Alfieri C, Vettoretti S. Metabolic acidosis in renal transplantation: neglected but of potential clinical relevance. Nephrol Dial Transplant. 2016 May;31(5):730-6.

2 Yakupoglu HY, Corsenca A, Wahl P, Wüthrich RP, Ambühl PM. Posttransplant acidosis and associated disorders of mineral metabolism in patients with a renal graft. Transplantation. 2007 Nov;84(9):1151-7.

3 Kocyigit I, Unal A, Kavuncuoglu F, Sipahioglu MH, Tokgoz B, Oymak 0, et al. Renal tubular acidosis in renal transplantation recipients. Ren Fail. 2010 Jul;32(6):687-90.

4 Keven K, Ozturk R, Sengul S, Kutlay S, Ergun I, Erturk S, et al. Renal tubular acidosis after kidney transplantation-incidence, risk factors and clinical implications. Nephrol Dial Transplant. 2007 Mar;22(3):906-10.

5 Ambühl PM. Posttransplant metabolic acidosis: a neglected factor in renal transplantation? Curr Opin Nephrol Hypertens. 2007 Jul;16(4):379-87.

6 Kraut JA, Madias NE. Metabolic Acidosis of CKD: an Update. Am J Kidney Dis. 2016 Feb;67(2):307-17.

7 Kraut JA, Madias NE. Adverse Effects of the Metabolic Acidosis of Chronic Kidney Disease. Adv Chronic Kidney Dis. 2017 Sep;24(5):289-97.

8 Kopple JD, Kalantar-Zadeh K, Mehrotra R. Risks of chronic metabolic acidosis in patients with chronic kidney disease. Kidney Int Suppl. 2005 Jun;67(95):S21-7.

9 Starke A, Corsenca A, Kohler T, Knubben J, Kraenzlin M, Uebelhart D, et al. Correction of metabolic acidosis with potassium citrate in renal transplant patients and its effect on bone quality. Clin J Am Soc Nephrol. 2012 Sep;7(9):1461-72.

10 Kovesdy CP, Anderson JE, Kalantar-Zadeh K. Association of serum bicarbonate levels with mortality in patients with non-dialysis-dependent CKD. Nephrol Dial Transplant. 2009 Apr;24(4):1232-7.

11 Phisitkul S, Khanna A, Simoni J, Broglio K, Sheather S, Rajab MH, et al. Amelioration of metabolic acidosis in patients with low GFR reduced kidney endothelin production and kidney injury, and better preserved GFR. Kidney Int. 2010 Apr;77(7):617-23.

12 Mahajan A, Simoni J, Sheather SJ, Broglio KR, Rajab MH, Wesson DE. Daily oral sodium bicarbonate preserves glomerular filtration rate by slowing its decline in early hypertensive nephropathy. Kidney Int. 2010 Aug; 78(3):303-9.

13 de-Brito Ashurst I, O’Lone E, Kaushik T, McCafferty K, Yaqoob MM. Acidosis: progression of chronic kidney disease and quality of life. Pediatr Nephrol. 2015 Jun;30(6):873-9.

14 Driver TH, Shlipak MG, Katz R, Goldenstein L, Sarnak MJ, Hoofnagle AN, et al. Low serum bicarbonate and kidney function decline: the Multi-Ethnic Study of Atherosclerosis (MESA). Am J Kidney Dis. 2014 Oct;64(4): 534-41.

15 Goldenstein L, Driver TH, Fried LF, Rifkin DE, Patel KV, Yenchek RH, et al.; Health ABC Study Investigators. Serum bicarbonate concentrations and kidney disease progression in community-living elders: the Health, Aging, and Body Composition (Health ABC) Study. Am J Kidney Dis. 2014 Oct;64(4): 542-9.

16 de Brito-Ashurst I, Varagunam M, Raftery MJ, Yaqoob MM. Bicarbonate supplementation slows progression of CKD and improves nutritional status. J Am Soc Nephrol. 2009 Sep;20(9):2075-84.

17 Goraya N, Wesson DE. Does correction of metabolic acidosis slow chronic kidney disease progression? Curr Opin Nephrol Hypertens. 2013 Mar;22(2):193-7.

18 Dobre M, Rahman M, Hostetter TH. Current status of bicarbonate in CKD. J Am Soc Nephrol. 2015 Mar;26(3): 515-23.

19 Goraya N, Simoni J, Jo CH, Wesson DE. A comparison of treating metabolic acidosis in CKD stage 4 hypertensive kidney disease with fruits and vegetables or sodium bicarbonate. Clin J Am Soc Nephrol. 2013 Mar;8(3):37181.

20 Park S, Kang E, Park S, Kim YC, Han SS, Ha J, et al. Metabolic Acidosis and Long-Term Clinical Outcomes in Kidney Transplant Recipients. J Am Soc Nephrol. 2017 Jun;28(6):1886-97.

21 Bonani M, Frey D, Brockmann J, Fehr T, Mueller TF, Saleh L, et al. Effect of Twice-Yearly Denosumab on Prevention of Bone Mineral Density Loss in De Novo Kidney Transplant Recipients: A Randomized Controlled Trial. Am J Transplant. 2016 Jun;16(6):1882-91.

22 Bonani M, Rodriguez D, Fehr T, Mohebbi N, Brockmann J, Blum M, et al. Sclerostin blood levels before and after kidney transplantation. Kidney Blood Press Res. 2014;39(4):230-9.

23 Pinheiro J BD, DebRoy S, Sarkar D and R Core Team: nlme: Linear and Nonlinear Mixed Effects Models. R package. version 3. 2006. p. 1-137.

24 Pilch NA, Rohan V, Rao V, Mauldin PD, Su Z, Dubay DA, et al. Renal Function Variability: An Independent Risk Factor for Graft Loss and Death following Kidney Transplantation. Am J Nephrol. 2018;47(3):191-9.

25 Choi HY, Huh KH, Lee JG, Song MK, Kim MS, Kim YS, et al. Variability of the Estimated Glomerular Filtration Rate in the First Year after Kidney Transplantation Is an Independent Risk Factor for Poor Renal Allograft Outcomes: A Retrospective Cohort Study. PLoS One. 2016 Dec;11(12):e0168337.

26 Xie Y, Bowe B, Xian H, Balasubramanian S, Al-Aly Z. Estimated GFR Trajectories of People Entering CKD Stage 4 and Subsequent Kidney Disease Outcomes and Mortality. Am J Kidney Dis. 2016 Aug;68(2):21928.

27 Hariharan S, McBride MA, Cherikh WS, Tolleris CB, Bresnahan BA, Johnson CP. Post-transplant renal function in the first year predicts long-term kidney transplant survival. Kidney Int. 2002 Jul;62(1):311-8. 
28 Kasiske BL, Israni AK, Snyder JJ, Skeans MA; Patient Outcomes in Renal Transplantation (PORT) Investigators. The relationship between kidney function and long-term graft survival after kidney transplant. Am J Kidney Dis. 2011 Mar; $57(3): 466-75$.

29 Salvadori M, Rosati A, Bock A, Chapman J, Dussol B, Fritsche L, et al. One-year posttransplant renal function is a strong predictor of long-term kidney function: results from the Neoral-MOST Observational Study. Transplant Proc. 2003 Dec;35(8):2863-7.

30 Choi HY, Joo DJ, Song MK, Kim MS, Park HC, Kim YS, et al. The Power of Renal Function Estimation Equations for Predicting Long-Term Kidney Graft Survival: A Retrospective Comparison of the Chronic Kidney Disease Epidemiology Collaboration and the Modification of Diet in Renal Disease Study Equations. Medicine (Baltimore). 2016 Feb;95(7):e2682.

31 First MR. Renal function as a predictor of long-term graft survival in renal transplant patients. Nephrol Dial Transplant. 2003 May;18(90001 Suppl 1):i3-6.

32 Lenihan CR, O'Kelly P, Mohan P, Little D, Walshe JJ, Kieran NE, et al. MDRD-estimated GFR at one year postrenal transplant is a predictor of long-term graft function. Ren Fail. 2008;30(4):345-52.

33 Lam NN, Tonelli M, Lentine KL, Hemmelgarn B, Ye F, Wen K, et al. Albuminuria and posttransplant chronic kidney disease stage predict transplant outcomes. Kidney Int. 2017 Aug;92(2):470-8. 